Corresponding author: Radomir I. Slavchov, Department of Chemical Engineering and Biotechnology,

Cambridge University, Pembroke Street, New Museums Site, UK, CB2 3RA Cambridge phone: 07787-468441; e-mail: ris26@cam.ac.uk

\title{
Contribution of the Surface Dipole Moment and the Contact Potential-Induced Disjoining Pressure to the Stress Balance at a Three-Phase Contact
}

\author{
Radomir I. Slavchov ${ }^{1,2,}$, Iglika M. Dimitrova ${ }^{2}$, Boryan P. Radoev ${ }^{2}$ \\ ${ }^{1}$ Department of Chemical Engineering and Biotechnology, Cambridge University, \\ UK, CB2 3RA Cambridge \\ ${ }^{2}$ Department of Physical Chemistry, Faculty of Chemistry and Pharmacy, Sofia University, 1164 Sofia, \\ Bulgaria \\ *ris26@cam.ac.uk
}

\begin{abstract}
The contact between three insulators results in a set up of contact potentials related to the adsorbed dipole moment at each surface. The produced electric field applies force (disjoining pressure) on each interface. This disjoining pressure is long-ranged $\left(1 /\right.$ distance $\left.^{2}\right)$, it is proportional to the difference between the dielectric permittivities of the phases on the two sides of the interface and, for small angles, to the square of the contact angle. The contact potential leads to a logarithmic perturbation of the profile of the threephase contact zone.
\end{abstract}

Keywords: surface potential, three-phase contact angle, wetting, insulator 


\section{CONTACT POTENTIAL IN A WEDGE}

Every interface possesses "adsorbed" normal dipole moment (Gibbs excess of the bulk polarization) due to the inherent anisotropy of the interfacial layer - in essence, this layer is a pyroelectric material [1]. The surface normal dipole results in a normal jump of the electrostatic potential [2] between each two macroscopic phases. This jump is called the contact potential [3]. The contact potential leads to no experimentally observable consequences in systems were there is a single homogeneously polarized surface (e.g., a spherical droplet). However, an inhomogeneity of the surface can cause a measurable gradient of the dipolar potential and a macroscopic electric field (e.g. [4]). Macroscopic field occurs also in the case where two different homogeneous interfaces are in contact with each other [3,5].

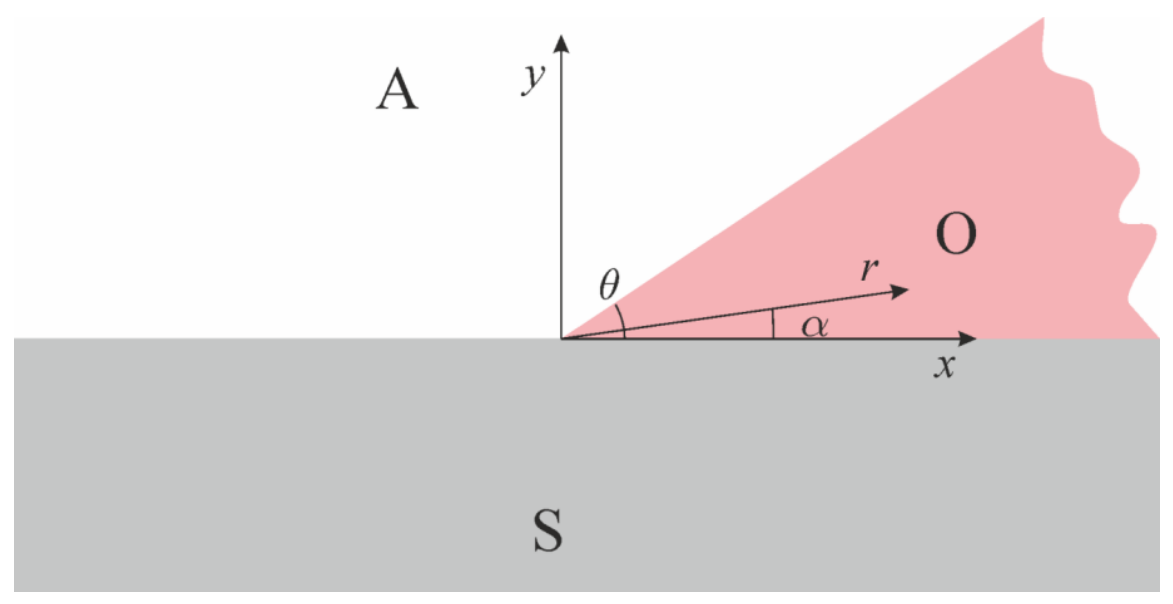

Fig. 1. Wedge geometry.

Here, we will investigate the macroscopic field in a wedge-shaped three-phase contact (TPC) zone between insulators - a solid substrate (S), and two fluids, say oil and air (O and A), with the geometry given in Fig. 1 ( $\operatorname{polar}_{[r, \alpha]} \operatorname{coordinate~system).~This~is~a~fairly~}$ common configuration in nature and technology, e.g. [6]. At each interface, a potential jump occurs due to the adsorbed dipole moment [2,3] - the three potential jumps are

$$
\Delta_{\mathrm{S}}^{\mathrm{O}} \phi=\left.\phi^{\mathrm{O}}\right|_{\alpha=0}-\left.\phi^{\mathrm{S}}\right|_{\alpha=2 \pi}, \quad \Delta_{\mathrm{S}}^{\mathrm{A}} \phi=\left.\left(\phi^{\mathrm{A}}-\phi^{\mathrm{S}}\right)\right|_{\alpha=\pi} \quad \text { and } \quad \Delta_{\mathrm{A}}^{\mathrm{O}} \phi=\left.\left(\phi^{\mathrm{O}}-\phi^{\mathrm{A}}\right)\right|_{\alpha=\theta},
$$

where $\phi^{\mathrm{X}}$ is the potential in the phase $\mathrm{X}, \theta$ is the TPC angle, and the jump $\Delta_{\mathrm{X}}^{\mathrm{Y}} \phi$ is a coordinate-independent characteristic of the interface $\mathrm{X} \mid \mathrm{Y}$ (the surface excess of the normal 
polarization divided by the vacuum permittivity [3]). The contact potentials $\Delta_{\mathrm{X}}^{\mathrm{Y}} \phi$ result in a field that applies ponderomotoric force to the interfaces. Our task in this work is, first, to determine the relationship between the force (the disjoining pressure [7]) acting in the wedge and the potentials $\Delta_{\mathrm{X}}^{\mathrm{Y}} \phi$, and second, to analyse their effect on the shape of the TPC zone of an insulating droplet at the surface of an insulating solid.

Eqs (1) are three boundary conditions for Coulomb's law of electrostatics. The other three are the conditions for continuity of the normal displacement field:

$$
\left.\varepsilon^{\mathrm{O}} E_{\alpha}^{\mathrm{O}}\right|_{\alpha=0}-\left.\varepsilon^{\mathrm{S}} E_{\alpha}^{\mathrm{S}}\right|_{\alpha=2 \pi}=0, \quad \varepsilon^{\mathrm{A}} E_{\alpha}^{\mathrm{A}}-\left.\varepsilon^{\mathrm{S}} E_{\alpha}^{\mathrm{S}}\right|_{\alpha=\pi}=0 \text { and } \varepsilon^{\mathrm{O}} E_{\alpha}^{\mathrm{O}}-\left.\varepsilon^{\mathrm{A}} E_{\alpha}^{\mathrm{A}}\right|_{\alpha=\theta}=0,
$$

where $E_{\alpha}=r^{-1} \mathrm{~d} \phi / \mathrm{d} \alpha$ is the $\alpha$-component of the electric field intensity and $\varepsilon$ are the dielectric permittivities of the phases.

The solution to Coulomb's equation in each phase $\left(\nabla^{2} \phi^{\mathrm{O}}=0, \nabla^{2} \phi^{\mathrm{A}}=0, \nabla^{2} \phi^{\mathrm{S}}=0\right)$ for the considered geometry reads (compare to Landau and Lifshitz [3]):

$$
\begin{aligned}
& \phi^{\mathrm{O}}=\phi_{0}^{\mathrm{O}}-e^{\mathrm{O}} \alpha / \varepsilon^{\mathrm{O}}, \quad \phi^{\mathrm{A}}=\phi_{0}^{\mathrm{A}}-e^{\mathrm{A}} \alpha / \varepsilon^{\mathrm{A}} \quad \text { and } \quad \phi^{\mathrm{S}}=\phi_{0}^{\mathrm{S}}-e^{\mathrm{S}} \alpha / \varepsilon^{\mathrm{S}} ; \\
& E_{\alpha}^{\mathrm{O}}=e^{\mathrm{O}} / \varepsilon^{\mathrm{O}} r, \quad E_{\alpha}^{\mathrm{A}}=e^{\mathrm{A}} / \varepsilon^{\mathrm{A}} r, \quad E_{\alpha}^{\mathrm{S}}=e^{\mathrm{S}} / \varepsilon^{\mathrm{S}} r .
\end{aligned}
$$

Here, $\phi_{0}$ and $e$ are six integration constants determined by the boundary conditions (1)\&(2) and by the arbitrary choice of the zero of the potential $\phi$. We set the zero of $\phi$ in the solid phase, right next to the $\mathrm{S} \mid \mathrm{O}$ interface, i.e. $\phi^{\mathrm{S}}(2 \pi)=0$. This condition, together with Eqs (1) $\&(2)$, leads to the following expressions for the integration constants in (3):

$$
\begin{aligned}
& e^{\mathrm{O}}=e^{\mathrm{A}}=e^{\mathrm{S}}=\frac{1}{\pi\left(1 / \varepsilon^{\mathrm{A}}+1 / \varepsilon^{\mathrm{S}}\right)} \frac{\Delta \phi}{1-\theta / \Theta} \quad(\equiv e) ; \\
& \phi_{0}^{\mathrm{O}}=\Delta_{\mathrm{S}}^{\mathrm{O}} \phi, \quad \phi_{0}^{\mathrm{A}}=\Delta_{\mathrm{S}}^{\mathrm{A}} \phi+\frac{\Delta \phi}{1-\theta / \Theta}, \quad \phi_{0}^{\mathrm{S}}=\frac{2}{1+\varepsilon^{\mathrm{S}} / \varepsilon^{\mathrm{A}}} \frac{\Delta \phi}{1-\theta / \Theta} .
\end{aligned}
$$

Here, we introduced the symbols

$$
\Delta \phi=\Delta_{\mathrm{S}}^{\mathrm{O}} \phi-\Delta_{\mathrm{A}}^{\mathrm{O}} \phi-\Delta_{\mathrm{S}}^{\mathrm{A}} \phi \quad \text { and } \quad \Theta=\frac{1+\varepsilon^{\mathrm{A}} / \varepsilon^{\mathrm{S}}}{1-\varepsilon^{\mathrm{A}} / \varepsilon^{\mathrm{O}}} \pi,
$$

to which we will refer as to the TPC potential $\Delta \phi$ and the dielectric ratio $\Theta$.

The following properties of the electric field in the wedge can be inferred from Eqs (4)-(6):

(i) from Eqs (4) it follows that the fields $E_{\alpha}^{\mathrm{O}}, E_{\alpha}^{\mathrm{A}}$ and $E_{\alpha}^{\mathrm{S}}$ act in the same direction, i.e. the $e$ 's in the three phases are of the same sign. The direction of the field (clockwise or 3 
anticlockwise) is controlled by the sign of the TPC potential $\Delta \phi$, as the denominator in Eqs (4) is always positive. When $\Delta \phi=0$, field is absent.

(ii) There is a monotonic dependence of $E_{\alpha}$ on $\theta$ : the field intensity in all phases increases as the contact angle $\theta$ increases if $\varepsilon^{\mathrm{O}}>\varepsilon^{\mathrm{A}}$, and vice versa.

(iii) The electric displacement is independent of $\alpha$ and is equal in all phases $\left(D_{\alpha}^{\mathrm{X}}=e^{\mathrm{X}} / r\right.$ and $e^{\mathrm{O}}=e^{\mathrm{A}}=e^{\mathrm{S}}$ ).

The solution (3) for the potential in the wedge is plotted in Fig. 2 as a function of the polar angle $\alpha$, for the following values of the parameters: $\varepsilon^{\mathrm{O}}=4 \times \varepsilon_{0}, \varepsilon^{\mathrm{A}}=\varepsilon_{0}, \varepsilon^{\mathrm{S}}=3 \times \varepsilon_{0}, \Delta_{\mathrm{S}}^{\mathrm{O}} \phi=$ $0.4 \mathrm{~V}, \Delta_{\mathrm{S}}^{\mathrm{A}} \phi=0.3 \mathrm{~V}, \Delta_{\mathrm{A}}^{\mathrm{O}} \phi=-0.4 \mathrm{~V}, \theta=60^{\circ}$.

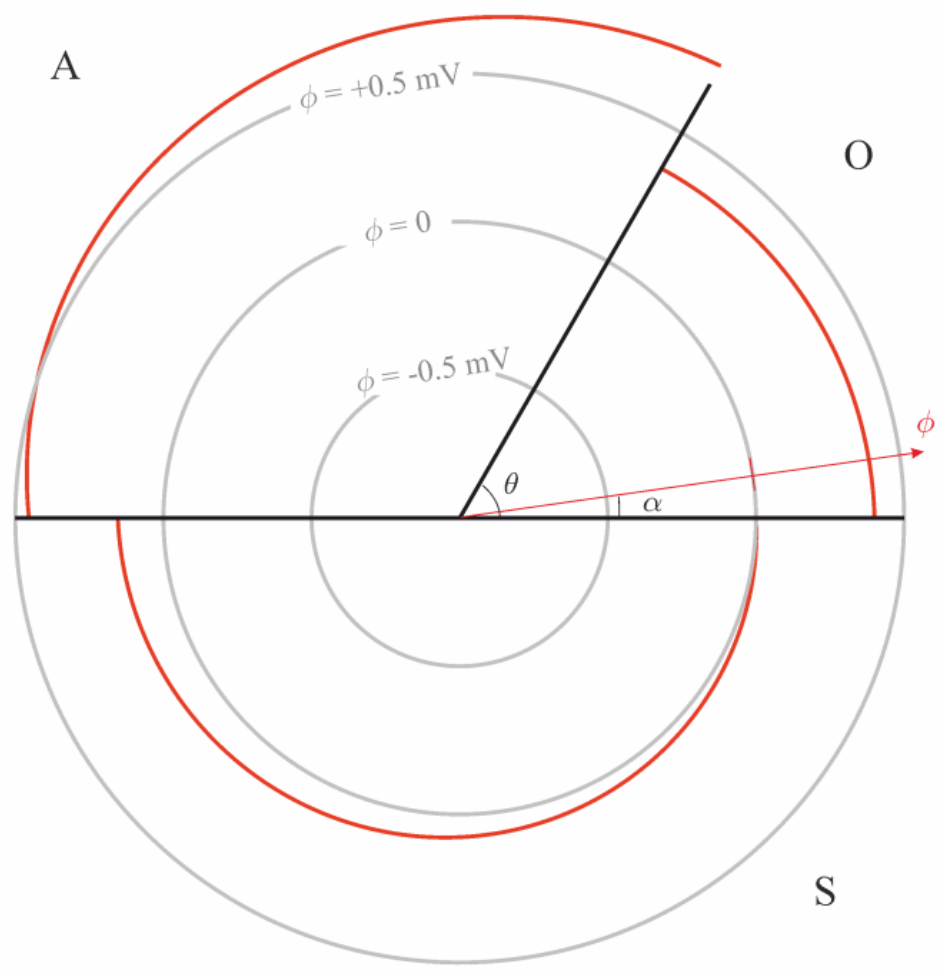

Fig. 2. Potential distribution in a wedge, $\phi$ vs. $\alpha$, according to Eqs (3)-(5). The circle $\phi=$ 0 is used as an abscissa.

Let us stress here that the contact potential is normally a dynamic phenomenon [3]. However small, the conductivity of the materials will eventually cause charge accumulation and formation of double layers that screen the contact potentials. For good insulators, both the relaxation time and the thickness of the double layer can be significant, and in the limit of perfect insulators, the results refer to equilibrium. 


\section{MAXWELL STRESS TENSOR}

We will now consider the ponderomotoric forces acting in the wedge. We skip the phase superscripts $\mathrm{O}, \mathrm{A}$ and $\mathrm{S}$ in this section for clarity, except where needed. The Maxwell stress tensor in each phase is given by [2]

$$
\boldsymbol{T}_{E}=\boldsymbol{D} \boldsymbol{E}-\frac{E^{2}}{2}\left(\varepsilon-C \frac{\partial \varepsilon}{\partial C}\right) \mathbf{U}=-\frac{E_{\alpha}^{2}}{2}\left(\begin{array}{ccc}
\varepsilon-C \frac{\partial \varepsilon}{\partial C} & 0 & 0 \\
& -\varepsilon-C \frac{\partial \varepsilon}{\partial C} & 0 \\
& & \varepsilon-C \frac{\partial \varepsilon}{\partial C}
\end{array}\right),
$$

where $\mathbf{U}$ is the unit tensor and $C$ is the density of the medium; cylindrical $[r, \alpha, z]$ coordinates are used. The total stress tensor $\boldsymbol{T}$ involves also the mechanical pressure $p$ :

$$
\boldsymbol{T}=-p(r, \alpha) \mathbf{U}+\boldsymbol{T}_{E} .
$$

The mechanical balance in each phase reads:

$$
\nabla \cdot \boldsymbol{T}=\mathbf{e}_{r} \frac{\partial T_{r r}}{\partial r}+\mathbf{e}_{\alpha} \frac{1}{r} \frac{\partial T_{\alpha \alpha}}{\partial \alpha}+\mathbf{e}_{z} \frac{\partial T_{z z}}{\partial z}=0,
$$

where $\mathbf{e}$ are the unit vectors. From the $\alpha$-component of this vectorial equation and the expression for the component $T_{\alpha \alpha}$ that follows from Eqs (3)\&(7)-(8) it follows that the mechanical pressure is independent of $\alpha$, i.e. $p=p(r)$. Then, the $r$-component of (9) yields

$$
\left(T_{r r}=\right)-p(r)-\frac{e^{2}}{2 \varepsilon^{2} r^{2}}\left(\varepsilon-C \frac{\partial \varepsilon}{\partial C}\right)=-p_{0}(\alpha),
$$

where $p_{0}$ is an $r$-independent integration constant. The expression of the left hand side of this equation is independent of $\alpha$, and the one on the right is independent of $r$, so both are constants, i.e.

$$
p(r)=p_{0}-\frac{e^{2}}{2 \varepsilon^{2} r^{2}}\left(\varepsilon-C \frac{\partial \varepsilon}{\partial C}\right) .
$$

By setting here $r \rightarrow \infty$, one can make the conclusion that $p_{0}$ is the pressure in the phases far away from the TPC line. At infinity the interfaces are flat, so $p_{0}$ is not phase-specific (Young-Laplace equation at $r \rightarrow \infty$ requires that $p_{0}^{\mathrm{O}}=p_{0}^{\mathrm{A}}=p_{0}^{\mathrm{S}}$ ).

Substituting the result (11) for $p$ in $\mathrm{Eq}$ (8) for $\boldsymbol{T}$, one obtains 


$$
\boldsymbol{T}=\left(\begin{array}{ccc}
-p_{0} & 0 & 0 \\
& -p_{0}+\varepsilon E_{\alpha}^{2} & 0 \\
& & -p_{0}
\end{array}\right) .
$$

From the definition (eq 5 of Rusanov and Shchekin [7]), we find that the disjoining pressure in each phase of the wedge is

$$
\Pi=-p_{0}-T_{\alpha \alpha}=-e^{2} / \varepsilon r^{2} .
$$

The expressions (12)\&(13) allow us to calculate the forces acting on each interface in Fig. 1. Let us summarize the most interesting properties of the disjoining pressure:

(i) $\Pi$ in each phase is attractive. The direction of the deformation of the $\mathrm{O} \mid \mathrm{A}$ interface is determined by the more attractive pressure of $\Pi^{\mathrm{O}}$ and $\Pi^{\mathrm{A}}$.

(ii) As $e^{\mathrm{O}}=e^{\mathrm{A}}=e^{\mathrm{S}}$, the ratio between the disjoining pressures in the three phases is:

$$
\Pi^{\mathrm{O}}: \Pi^{\mathrm{A}}: \Pi^{\mathrm{P}}=1 / \varepsilon^{\mathrm{O}}: 1 / \varepsilon^{\mathrm{A}}: 1 / \varepsilon^{\mathrm{S}} \text {. }
$$

The most attractive is the disjoining pressure in the least polar phase - for the considered case, this is the air. Therefore, for the configuration in Fig. 1, the electrostatic interactions will pull the interface toward the gas phase.

(iii) The considered disjoining pressure is long ranged - by expressing Eq (13) with the "distance" $y=r \sin (\theta)$ between the solid surface and the O|A interface, one obtains:

$$
\Pi^{\mathrm{O}}=-\frac{e^{2}}{\varepsilon^{\mathrm{O}}} \frac{\sin ^{2} \theta}{y^{2}},
$$

i.e., $\Pi \propto y^{-2}$. Thus, the force due to the contact potential in insulators is more long-ranged than the van der Waals attraction $\left(\Pi \propto y^{-3}\right)$.

Let us now consider the limitations of the derived expressions. Near the TPC, the solution for the electrostatic potential becomes unphysical - the obtained results (3) for the electric field and (13) for $\Pi$ are singular at the origin. The distance at which Eqs (3)\&(13) become invalid can be estimated via the analysis of the approximations that are responsible for the singularity. The first omitted factor is the finite thickness of the polarized surface layer (the diffuse dipolar layer [1]). The characteristic length of the dipole layer is the socalled quadrupolar length $L_{Q}$ [1], which measures the ratio between the quadrupolar and the dipolar strengths of the insulator (the quadrupolarizability of the medium is important for the structure of the dipole layer due to the conjugation between surface dipole and bulk 
quadrupolarization [8]). The order of magnitude of $L_{Q}$ for oils is $\sim \AA$ [9]; thus, the result (13) becomes invalid at $r$ of the order of a few $\AA$.

A second limitation of Eq (13) is related to the change of the polarization of the surfaces near the TPC. The electric field (3) tends to infinity at $r=0$ so near the TPC it is large enough to alter $\Delta_{\mathrm{S}}^{\mathrm{O}} \phi, \Delta_{\mathrm{S}}^{\mathrm{A}} \phi \& \Delta_{\mathrm{A}}^{\mathrm{O}} \phi$ (i.e. close enough to the TPC, the short-ranged "strong" orienting molecular image force that causes the polarization of each surface [10] and the long-ranged "weak" interaction between the three dipolar surfaces are of similar order of magnitude). Thus, the assumed independence of $r$ of the contact potentials must fail at certain distance from the TPC. To estimate this distance, we assume that a linear relationship between the contact potential and the normal displacement field $D_{\alpha}$ holds [1]:

$$
\Delta_{\mathrm{A}}^{\mathrm{O}} \phi\left(D_{\alpha}\right)=\Delta_{\mathrm{A}}^{\mathrm{O}} \phi\left(D_{\alpha}=0\right)+\chi^{\mathrm{S}} D_{\alpha} / \varepsilon_{0} .
$$

Here $D_{\alpha}(r)=\varepsilon E_{\alpha}=e / r$, and $\chi^{\mathrm{S}}$ is the dielectric susceptibility of the dipolar double layer. This quantity can be estimated using eq 68 from Ref. [1], which reads for the O|A interface:

$$
\chi^{\mathrm{S}}=\left(\frac{\varepsilon^{\mathrm{O}}}{\alpha_{z z}^{\mathrm{S}}}+\frac{\varepsilon^{\mathrm{O}}}{\varepsilon_{0} L_{Q}^{\mathrm{O}}}\right)^{-1} .
$$

Here, the intrinsic surface polarizability $\alpha_{z z}^{\mathrm{S}}$ is of the order of $\sim \varepsilon_{0} L_{Q}^{\mathrm{O}}$; using $L_{Q}^{\mathrm{O}}=2 \AA$ [9] and $\varepsilon^{\mathrm{O}}=4 \varepsilon_{0}$, one obtains $\chi^{\mathrm{S}} \sim 0.25 \AA$. The considered depolarization effect becomes significant when $\chi^{\mathrm{S}} D_{\alpha}$ is, e.g., $10 \%$ of $\varepsilon_{0} \Delta_{\mathrm{A}}^{\mathrm{O}} \phi$, or from Eqs (3)\&(4), when $r \sim 10 \times \chi^{\mathrm{S}} e / \varepsilon_{0} \Delta_{\mathrm{A}}^{\mathrm{O}} \phi$ $\sim 1 \AA$. Therefore, significant surface depolarization can be expected only in the immediate neighbourhood (1-2 molecules) of the TPC.

A third phenomenon we neglect that becomes important near the TPC is the dielectroMarangoni effect [1]: the effect of the normal field on the value of the mechanical [11] surface tension $\gamma$. For small fields, it follows from $E q 85$ in Ref. [1] that:

$$
\gamma=\sigma(r=\infty)+\chi^{\mathrm{s}} D_{\alpha}^{2} / 2 \varepsilon_{0}, \text { or } \mathrm{d} \gamma / \mathrm{d} r=-\chi^{\mathrm{s}} e^{2} / \varepsilon_{0} r^{3},
$$

where Eq (3) was used. The Marangoni force $\mathrm{d} \gamma / \mathrm{d} r$ becomes significant at about the same distance from the rim (a few $\AA$ ) as the previous two effects we discussed.

\section{DEFORMATION OF THE SURFACE AND DYNAMIC}


The two stress tensors acting on the two sides of the O|A surface are locally unbalanced $\left(\Delta T_{\alpha \alpha}=T_{\alpha \alpha}^{\mathrm{O}}-T_{\alpha \alpha}^{\mathrm{A}} \neq 0\right)$. The liquid interface will answer to the uncompensated force by deformation leading to corrugation, and respectively, a capillary pressure $p_{\mathrm{c}}=\sigma / R_{\mathrm{c}}$ balancing $\Delta T_{\alpha \alpha}\left(\sigma-\right.$ surface tension, $R_{\mathrm{c}}$ - radius of curvature). In this section, we will consider the problem for the profile or the TPC zone in three cases: (i) large angles, no van der Waals forces; (ii) small angles and van der Waals forces acting; (iii) the case of complete wetting and repulsive van der Waals force. In all cases, we assume that the capillary pressure far from the TPC is zero.

(i) The case of large TPC angle and absent van der Waals disjoining pressure. Voinov [12] solved a similar problem for the effect of the hydrodynamic flow in a wedge on the shape of a three-phase contact. Here, we repeat his analysis, with minor modifications and with electric instead of viscous stress tensor. Using the relation between the radius of curvature $R_{\mathrm{c}}$ and the shape $y(x)$ of the $\mathrm{O} \mid \mathrm{A}$ interface (in Cartesian $[x, y, z]$ coordinates, Fig. 1), we can write the balance between $\Delta T_{\alpha \alpha}(\mathrm{Eq}(12))$ and $p_{\mathrm{c}}$ as

$$
\sigma \frac{\operatorname{sg}\left(y_{x}\right) y_{x x}}{\left(1+y_{x}^{2}\right)^{3 / 2}}=-\Pi_{\Delta \phi}
$$

where $y_{x}$ and $y_{x x}$ are the first and the second derivative of $y(x), \mathrm{sg}$ is the signum function, and the disjoining pressure $\Pi_{\Delta \phi}$ stands for

$$
\Pi_{\Delta \phi}=-\Delta T_{\alpha \alpha}=\Pi^{\mathrm{O}}-\Pi^{\mathrm{A}}=-\left(\frac{1}{\varepsilon^{\mathrm{O}}}-\frac{1}{\varepsilon^{\mathrm{A}}}\right) \frac{e^{2}}{r^{2}} .
$$

Assuming that the local disjoining pressure is controlled by the local thickness of the liquid film $y$ and the local slope of the surface $y_{x}=\tan \theta$ (compare to [12]), we can rewrite Eq (19) as

$$
\frac{\operatorname{sg}\left(y_{x}\right) y_{x x}}{\left(1+y_{x}^{2}\right)^{3 / 2}}=-\left(1-\frac{\arctan y_{x}}{\Theta}\right)^{-2} \frac{L_{\Delta \phi}}{y^{2}} \frac{y_{x}^{2}}{1+y_{x}^{2}},
$$

where we used Eq (4) for $e$, the relation $r^{2}=\left(1+1 / y_{x}^{2}\right) \times y^{2}$, and we introduced the contact potential length:

$$
L_{\Delta \phi}=-\frac{1 / \varepsilon^{\mathrm{O}}-1 / \varepsilon^{\mathrm{A}}}{\left(1 / \varepsilon^{\mathrm{A}}+1 / \varepsilon^{\mathrm{S}}\right)^{2}} \frac{(\Delta \phi)^{2}}{\pi^{2} \sigma} .
$$


For small deviation $\delta y$ of the surface from the flat shape $y=x \tan \theta_{\infty}$, Eq (19) can be linearized with respect to $\delta y$ :

$$
\frac{\mathrm{d}^{2} \delta y}{\mathrm{~d} x^{2}}=-\frac{L_{\Delta \phi}}{\cos \theta_{\infty}\left(1-\theta_{\infty} / \Theta\right)^{2}} \frac{1}{x^{2}} .
$$

Double integration leads to the asymptotic solution for the shape of the O|A interface:

$$
y+\delta y=x \tan \theta_{\infty}+\frac{L_{\Delta \phi}}{\cos \theta_{\infty}\left(1-\theta_{\infty} / \Theta\right)^{2}} \ln \left|\frac{x}{x_{0}}\right|+\mathrm{O}\left(L_{\Delta \phi}^{2} x^{-1} \ln x\right),
$$

where $x_{0}$ is an integration constant (which is unimportant for the final results). The deviation term in $\mathrm{Eq}(19)$ is relatively small: the ratio $\delta y / y$ is significant only when $x \sim L_{\Delta \phi}$, which is of the order of $\AA$.

Let us also consider briefly the "exact" solution of Eq (19), with the reservation that it is not significantly more accurate than Eq (19), as the expression used in Laplace-Young equation (19) for the disjoining pressure is valid neither for large deviations from the flat shape, nor for distances of the order of a few $\AA$ (which is where the "exact" solution differs from $\mathrm{Eq}(19))$. A first integral of Eq (19) can be obtained by substituting $y_{x}=\tan \theta$ in it:

$$
\sin \theta \frac{\mathrm{d} \theta}{\mathrm{d} y}=-\frac{L_{\Delta \phi}}{y^{2}} \frac{\sin ^{2} \theta}{(1-\theta / \Theta)^{2}}
$$

which is integrated to

$$
\frac{L_{\Delta \phi}}{y}=\int_{\theta_{\infty}}^{\theta} \frac{(1-\theta / \Theta)^{2}}{\sin \theta} \mathrm{d} \theta
$$

We used as a boundary condition that $y \rightarrow \infty$ when $\theta \rightarrow \theta_{\infty}$, where $\theta_{\infty}$ is the macroscopic TPC angle far from the TPC zone. At the TPC contact $(y \rightarrow 0)$, the integral on the right hand side must be infinite, which means that $\theta\left(y=0\right.$ ) is either equal to $180^{\circ}$ (if $L_{\Delta \phi}>0$ ) or to $0^{\circ}$ (if $L_{\Delta \phi}<0$ ). The dependence of $\theta$ on $y$ that follows from Eq (19) is illustrated in Fig. $3(\sigma=20 \mathrm{mN} / \mathrm{m})$. However, as the formula (13) for $\Pi$ is invalid if the shape of the surface is too different from a wedge, Eq (19) should give the correct asymtote at $\theta-\theta_{\infty} \rightarrow 0$ but it becomes inaccurate as this difference increases. 


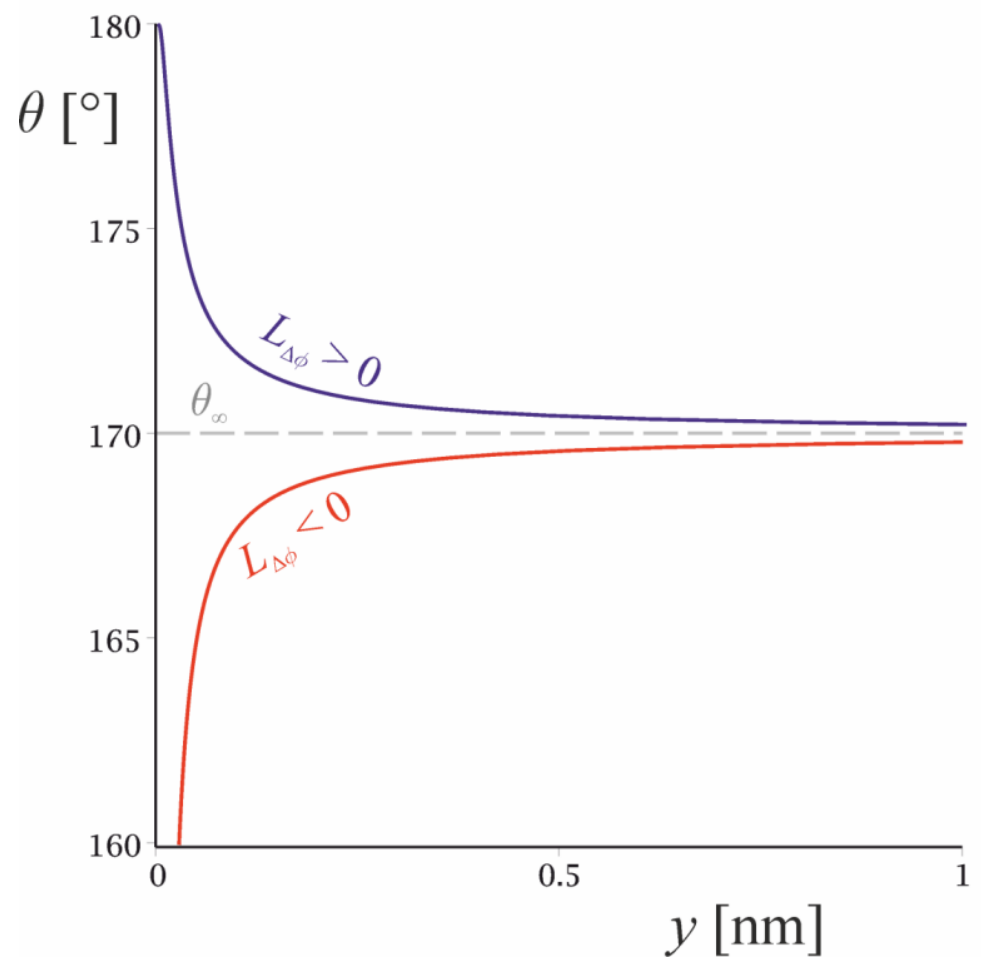

Fig. 3. Local slope angle $\theta=\arctan y_{x}$ of the liquid interface as a function of the distance $y$ between the solid and the liquid surfaces according to Eq (19). The upper curve is calculated with contact potential length $L_{\Delta \phi}=0.1 \AA$ (following from the parameter values in the text) and macroscopic angle $\theta_{\infty}=170^{\circ}$; the lower stands for $L_{\Delta \phi}=-0.1 \AA$.

Second integration of Eq (19) yields the $x$ coordinate; the easiest way to obtain it is to use the relation $x=\int \cot \theta \mathrm{d} y$; this integral is calculated numerically using the recurrent trapezoidal rule

$$
x_{i+1}=x_{i}+\left(\cot \theta_{i}+\cot \theta_{i+1}\right)\left(y_{i+1}-y_{i}\right) / 2 .
$$

Here, $y_{i}$ is the value following from $\mathrm{Eq}(19)$ at $\theta=\theta_{i}$; for a list of values of $\theta_{i}, i=1 \ldots n$, the respective $y_{i}$ are calculated through $\mathrm{Eq}$ (19), and then $\mathrm{Eq}$ (19) is used to calculate the respective $x_{i}$ (starting from an arbitrary $x_{1}$ ). The result for $y(x)$ is plotted in Fig. 4 for two values of $\theta_{\infty}$, and is compared to its asymptote (19). The integration constants $\left(x_{0} \& x_{1}\right)$ are chosen manually. 

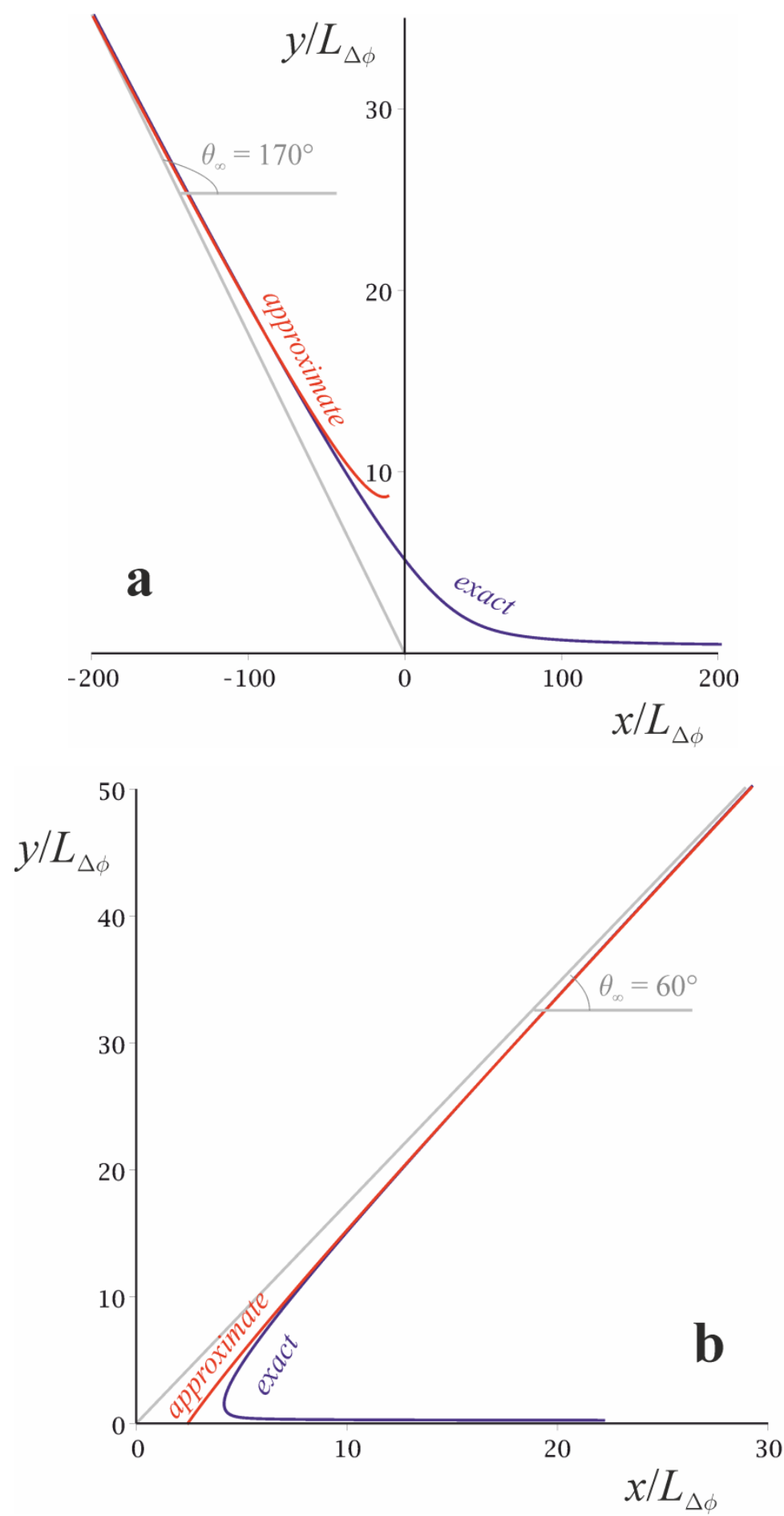

Fig. 4. Profile of the liquid interface for (a) $\theta_{\infty}=170^{\circ}$ and (b) $\theta_{\infty}=60^{\circ} ; x$ and $y$ are nondimensionalized with $L_{\Delta \phi}$. The curve labelled "exact" is calculated via Eqs (19)\&(19); the curve "approximate" corresponds to Eq (19). 
(ii) The case of small TPC angle and van der Waals force present. Now we will modify the Laplace-Young equation (19) in two aspects. First, we will assume small slopes and use the approximations $y_{x} \ll<1$ and $\theta_{\infty} \ll 1$. With this approximations, Eq (19) simplifies to $\Pi_{\Delta \phi}=\sigma L_{\Delta \phi} \times y_{x}^{2} / y^{2}$; thus, the contact potential-induced disjoining pressure $\Pi_{\Delta \phi}$ has an essential dependence on the slope, and vanishes for a plane parallel film. Second, we will account for the van der Waals disjoining pressure, which, for small slopes and neglected retardation effect, can be expressed as $\Pi_{\mathrm{vdW}}=-A_{\mathrm{H}} / 6 \pi y^{3}$ (where $A_{\mathrm{H}}$ is the Hamaker constant). The Laplace-Young-Derjaguin equation now reads:

$$
y_{x x}=-\frac{\Pi_{\Delta \phi}+\Pi_{\mathrm{vdW}}}{\sigma}=-L_{\Delta \phi} \frac{y_{x}^{2}}{y^{2}}+\frac{a_{\mathrm{vdW}}}{y^{3}},
$$

where we introduced the van der Waals effective area as

$$
a_{\mathrm{vdW}}=A_{\mathrm{H}} / 6 \pi \sigma \text {. }
$$

The constants $L_{\Delta \phi}$ and $a_{\mathrm{vdW}}$ in Eq (19) can have both positive and negative values $\left(\Pi_{\Delta \phi}\right.$ is repulsive if $L_{\Delta \phi}>0, \Pi_{\mathrm{vdW}}$ is attractive $a_{\mathrm{vdW}}>0$ ). For $\left|A_{\mathrm{H}}\right|>10^{-20} \mathrm{~J}$ and small TPC angles the $L_{\Delta \phi}$ term can be neglected and then Eq (19) simplifies to eq 2.48 in [13]. If $\left|A_{\mathrm{H}}\right|<10^{-21}$ $\mathrm{J}$, the contact potential becomes important, and in the limit of negligible van der Waals interaction, $\mathrm{Eq}(19)$ is a variant of $\mathrm{Eq}(19) . \mathrm{Eq}(19)$ has a first integral:

$$
y_{x}^{2}=\left(\theta_{\infty}^{2}-\frac{a_{\mathrm{vdW}}}{2 L_{\Delta \phi}^{2}}\right) \mathrm{e}^{2 L_{\Delta \phi} / y}+\frac{a_{\mathrm{vdW}}}{2 L_{\Delta \phi}^{2}}\left(1+\frac{2 L_{\Delta \phi}}{y}\right),
$$

where we used the boundary condition $(\mathrm{d} y / \mathrm{d} x)_{y=\infty}=\theta_{\infty}$. A second integration yields the profile of the TPC zone:

$$
x=\int\left[\left(\theta_{\infty}^{2}-\frac{a_{\mathrm{vdW}}}{2 L_{\Delta \phi}^{2}}\right) \mathrm{e}^{2 L_{\Delta \phi} / y}+\frac{a_{\mathrm{vdW}}}{2 L_{\Delta \phi}^{2}}\left(1+\frac{2 L_{\Delta \phi}}{y}\right)\right]^{-1 / 2} \mathrm{~d} y .
$$

Let us now analyse some limiting cases of this result. In the absence of contact potential $\left(L_{\Delta \phi}=0\right)$, the integration gives the well-known [13] result $y^{2}=\theta_{\infty}^{2} x^{2}+a_{\mathrm{vdW}} / \theta_{\infty}^{2}$. On the other hand, if van der Waals interaction is negligible $\left(a_{\mathrm{vdW}}=0\right)$, we reach:

$$
\theta_{\infty} x=y \mathrm{e}^{-L_{\Delta \phi} / y}-L_{\Delta \phi} \mathrm{E}_{1}\left(\frac{L_{\Delta \phi}}{y}\right) \stackrel{y \rightarrow \infty}{\longrightarrow} y-L_{\Delta \phi} \ln \frac{y}{\mathrm{e}^{\gamma-1}\left|L_{\Delta \phi}\right|}+\mathrm{O}\left(y^{-1}\right),
$$


where $E_{1}$ is the integral exponent of the first order (in case of $L_{\Delta \phi}<0-$ its real part) and $\gamma$ is Euler's constant; an arbitrary constant can be added to $x$. The same limit at $y \rightarrow \infty$ can be obtained from Eq (19) in the limit of small slopes. The series of Eq (19) at $y \rightarrow \infty$ if both van der Waals and contact potential-induced pressures are acting reads:

$$
\theta_{\infty} x \stackrel{y \rightarrow \infty}{\longrightarrow} y-L_{\Delta \phi} \ln \frac{y}{\mathrm{e}^{\gamma-1} L_{\Delta \phi}}-\left(\frac{L_{\Delta \phi}^{2}}{2}+\frac{a_{\mathrm{vdW}}}{2 \theta_{\infty}^{2}}\right) \frac{1}{y}+\mathrm{O}\left(\frac{1}{y^{2}}\right) .
$$

According to this result, the perturbation due to the contact potential of the profile from the flat shape is dominating over that due to the van der Waals force at large $y$, due to the fact that the contact potential-induced disjoining pressure is more long-ranged than the van der Waals $\left(1 / y^{2}\right.$ instead of $\left.1 / y^{3}\right)$.

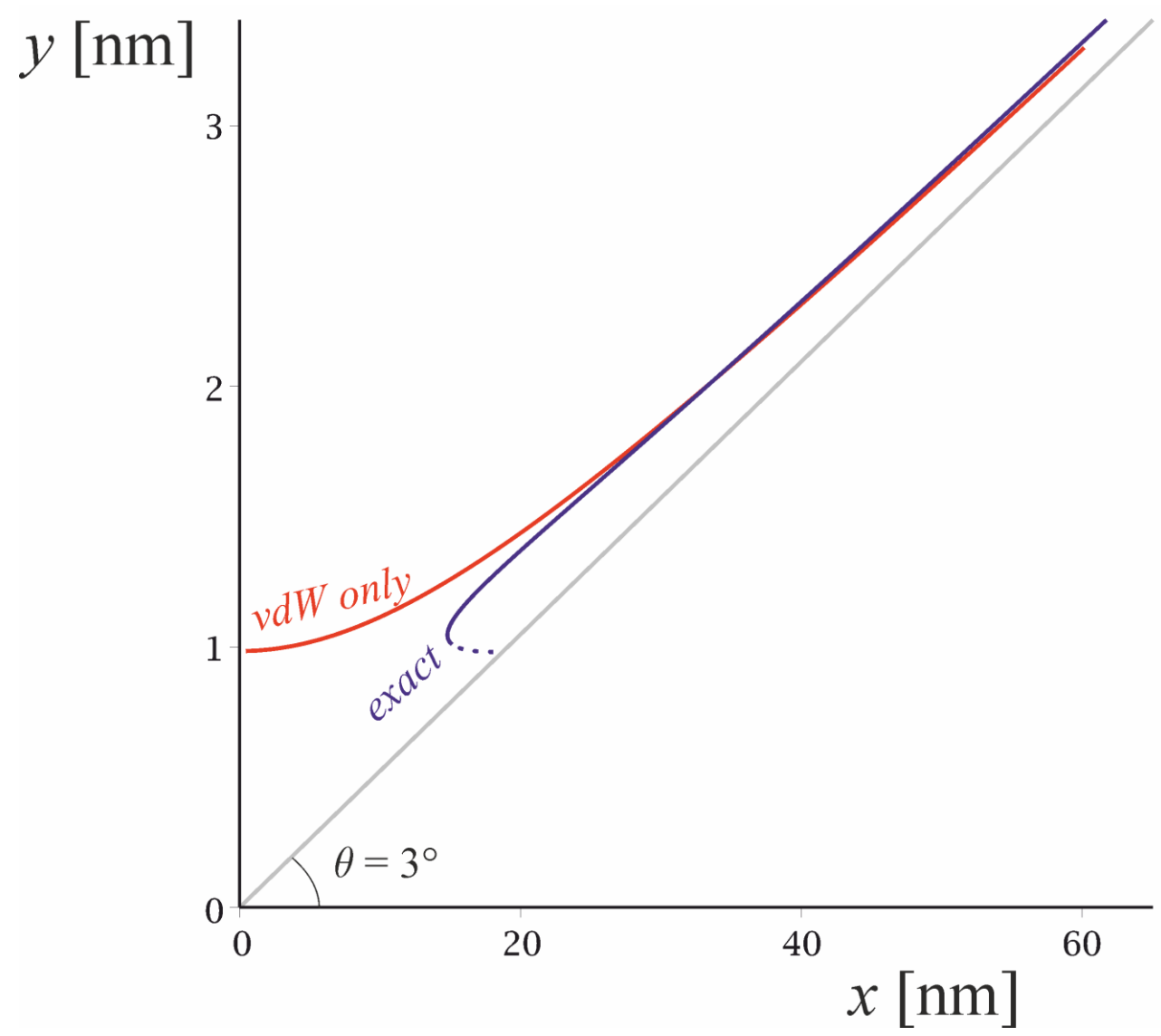

Fig. 5. Profile of the liquid interface for the case of small TPC angle $\left(\theta=3^{\circ}\right)$ in the presence of van der Waals attraction $\left(A_{\mathrm{H}}=10^{-21} \mathrm{~J}\right)$. The "vdW only" line corresponds to the known solution $y^{2}=\theta^{2} x^{2}+a_{\mathrm{vdW}} / \theta^{2}$ in the absence of contract potential. The line "exact" accounts also for the contact potential-induced disjoining pressure, Eq (19). 
The profile (19) is illustrated in Fig. 5 for the case where the van der Waals pressure is attractive $\left(A_{\mathrm{H}}=10^{-21} \mathrm{~J}\right)$ and the electrostatic parameters are as those above (repulsive $\left.\Pi_{\Delta \phi}\right)$. It is compared with the parabolic profile $y^{2}=\theta_{\infty}^{2} x^{2}+a_{\mathrm{vdw}} / \theta_{\infty}^{2}$ in the absence of contact potential; note that the two solutions differ both far from and close to the TPC line: far from it due to the logarithmic term $-L_{\Delta \phi} \operatorname{nn} y$ in $\mathrm{Eq}(19)$, and even more significantly close to it, where the curvature abruptly changes sign, and the slope increases. As Eq (19) is valid only for small slopes, the overhang (the extremum of $x(y)$ ) and the dotted part of the line in Fig. 5 are not physical. The qualitative result - that the repulsive disjoining pressure $\Pi_{\Delta \phi}$ limits the extent of the TPC zone - must be correct.

(ii) Complete wetting $\left(\theta_{\infty}=0\right)$. In the absence of capillary pressure, complete wetting is possible only if the van der Waals pressure is repulsive, $a_{\mathrm{vdW}}<0$. In this case, the profile near the TPC that follows from $\mathrm{Eq}(19)$ is:

$$
x=L_{\Delta \phi}^{2} \sqrt{\frac{2}{-a_{\mathrm{vdW}}}} \int\left[\mathrm{e}^{2 L_{\Delta \phi} / y}-1+\frac{2 L_{\Delta \phi}}{y}\right]^{-1 / 2} \mathrm{~d} \frac{y}{L_{\Delta \phi}} \approx \frac{y^{2}}{2 \sqrt{-a_{\mathrm{vdW}}}}\left(1-\frac{2 L_{\Delta \phi}}{3 y}\right),
$$

where the approximation is valid at $y>\left|L_{\Delta \phi}\right|$. This result simplifies to the known [13] asymptote $2\left(-a_{\mathrm{vdW}}\right)^{1 / 2} x=y^{2}$ when $y>\left|L_{\Delta \phi}\right|$. The effect from the contact potential is rather small in this case, since complete wetting corresponds to small slopes, and $\Pi_{\Delta \phi} \propto y_{x}^{2}$.

\section{CONCLUSIONS}

A contact potential arises in the TPC region between insulators, due to the dipole moment of the surfaces, that brings about a macroscopic electric field, Eq (3). The electric field is proportional to $1 / r$ and becomes significant in the vicinity of the TPC. We have shown that the respective Maxwell stress corresponds to a very long ranged contact potential-induced disjoining pressure $\Pi_{\Delta \phi}=\Pi^{P}-\Pi^{\mathrm{A}}$. The sign of $\Pi_{\Delta \phi}$ is controlled by the difference in the dielectric constants of the phases $\mathrm{O}$ and $\mathrm{A}$, and it is proportional to the square of the uncompensated TPC potential (6). This disjoining pressure is proportional 
to the square of the slope, and - within the validity of the macroscopic Maxwell equations - vanishes for flat films (more precisely, for flat films, $\Pi_{\Delta \phi}$ will be a short ranged force, acting at distances of the order of the thickness $L_{Q}$ of the diffuse dipolar layer [1]).

We have considered the displacement of the liquid interface in the TPC zone due to this new disjoining pressure for several physical cases (large angles, small angles and thin wetting films, with and without van der Waals forces). In all cases, the contact potentialinduced deformation is small but very long-ranged, even more than the one due to van der Waals interactions.

We limited our discussion to TPC between three insulators, but very similar expressions for the field and the disjoining pressure hold in the case where one or two of the phases are metals (these can be obtained, e.g., by setting $\varepsilon^{S} \rightarrow \infty$ in Eqs (4)-(6)), as well as for the case of a crystal edge, where two facets of different surface dipole moment join (by setting $\varepsilon^{\mathrm{S}}=\varepsilon^{\mathrm{A}}$ and $\Delta_{\mathrm{S}}^{\mathrm{A}} \phi=0$ ). 


\section{REFERENCES}

1. Slavchov, R.I., Dimitrova, I.M., and Ivanov, T., J. Chem. Phys. 2015, vol. 143, p. 154707.

2. Тамм, И.Е., Основы теории электричества, 10-е изд., Москва: Наука, 1989; гл. 2; Tamm, I.E., Fundamentals of the theory of electricity, 9th ed., Mir Publishers, 1979, Chap. 2.

3. Ландау, Л.Д. и Лифшиц, Е.М., Электродинамика сплошых сред, 2-е изд., Москва: Наука, 1982, гл. 3; Landau, L.D. and Lifshitz, E.M., Electrodynamics of continuous media, 2nd ed., Pergamon Press, 1984, Chap. 3.

4. Kochurova, N.N. and Rusanov, A.I., J. Colloid Interface Sci. 1981, vol. 81, p. 297.

5. McConnell, H., Annu. Rev. Phys. Chem. 1991, vol. 42, p. 171.

6. Yatsuzuka, K., Mizuno, Y., and Asano, K., J. Electrostatics, 1994, vol. 32, p. 157.

7. Rusanov, A.I. and Shchekin, A.K., Molecular Phys., 2005, vol. 103, p. 2911.

8. Батыгин, В.В. и Топтыгин, И.Н., Сборник задач по электродинамике и специиальной теории относительности, 4-е изд., ЛАН, 2010, с. 283.

9. Dimitrova, I.M., Slavchov, R.I., Ivanov, T., and Mosbach, S., J. Chem. Phys. 2016, vol. 144, p. 114502.

10. Френкель, Я., Кинетическая теория жидкостей, Ленинград: Наука, 1975, c. 398; Frenkel, J., Kinetic theory of liquids, New York: Dover Publications, 1955 , p. 356.

11. Rusanov, A.I. and Kuni, F.M., J. Colloid Interface Sci., 1984, vol. 100, p. 264.

12. Войнов, О.И., Изв. АН СССР: Механика жидкости газа, 1976, №5, с. 76;

Voinov, O.I., Fluid Dyn., 1976, vol. 11, p. 714.

13. De Gennes, P.G., Rev. Mod. Phys., 1985, vol. 57, p. 827. 